\title{
HATS-3b: AN INFLATED HOT JUPITER TRANSITING AN F-TYPE STAR*
}

\author{
D. Bayliss ${ }^{1}$, G. Zhou ${ }^{1}$, K. Penev ${ }^{2,3}$, G. Á. Bakos ${ }^{2,3,10,11}$, J. D. HARTman ${ }^{2,3}$, A. Jordán ${ }^{4}$, L. MANCINI $^{5}$, M. MohleR-Fischer ${ }^{5}$, \\ V. Suc ${ }^{4}$, M. Rabus ${ }^{4}$, B. Béky ${ }^{3}$, Z. Csubry ${ }^{2,3}$, L. Buchhave ${ }^{6}$, T. Henning ${ }^{5}$, N. Nikolov ${ }^{5}$, B. Csák $^{5}$, R. Brahm $^{4}$, N. Espinoza $^{4}$,

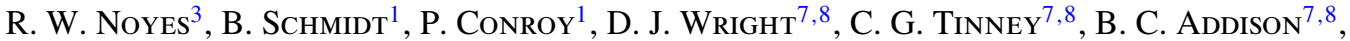 \\ P. D. Sackett ${ }^{1}$, D. D. Sasselov ${ }^{3}$, J. LÁzÁr $^{9}$, I. PaPP ${ }^{9}$, AND P. SÁri ${ }^{9}$ \\ ${ }^{1}$ Research School of Astronomy and Astrophysics, Australian National University, Canberra, ACT 2611, Australia; daniel@mso.anu.edu.au \\ ${ }^{2}$ Department of Astrophysical Sciences, Princeton University, NJ 08544, USA \\ ${ }^{3}$ Harvard-Smithsonian Center for Astrophysics, Cambridge, MA, USA \\ ${ }^{4}$ Departamento de Astronomía y Astrofísica, Pontificia Universidad Católica de Chile, \\ Av. Vicuña Mackenna 4860, 7820436 Macul, Santiago, Chile \\ ${ }^{5}$ Max Planck Institute for Astronomy, Heidelberg, Germany \\ ${ }^{6}$ Niels Bohr Institute, Copenhagen University, Denmark \\ ${ }^{7}$ Exoplanetary Science at UNSW, School of Physics, University of New South Wales, 2052, Australia \\ ${ }^{8}$ Australian Centre for Astrobiology, University of New South Wales, 2052, Australia \\ ${ }^{9}$ Hungarian Astronomical Association, Budapest, Hungary \\ Received 2013 June 3; accepted 2013 August 12; published 2013 September 27
}

\begin{abstract}
We report the discovery by the HATSouth survey of HATS-3b, a transiting extrasolar planet orbiting a $V=12.4 \mathrm{~F}$ dwarf star. HATS-3b has a period of $P=3.5479$ days, mass of $M_{p}=1.07 M_{\mathrm{J}}$, and radius of $R_{p}=1.38 R_{\mathrm{J}}$. Given the radius of the planet, the brightness of the host star, and the stellar rotational velocity $\left(v \sin i=9.0 \mathrm{~km} \mathrm{~s}{ }^{-1}\right)$, this system will make an interesting target for future observations to measure the Rossiter-McLaughlin effect and determine its spin-orbit alignment. We detail the low-/medium-resolution reconnaissance spectroscopy that we are now using to deal with large numbers of transiting planet candidates produced by the HATSouth survey. We show that this important step in discovering planets produces $\log g$ and $T_{\text {eff }}$ parameters at a precision suitable for efficient candidate vetting, as well as efficiently identifying stellar mass eclipsing binaries with radial velocity semi-amplitudes as low as $1 \mathrm{~km} \mathrm{~s}^{-1}$.
\end{abstract}

Key words: planetary systems - stars: individual (HATS-3, GSC 6926-00454) - techniques: photometric - techniques: spectroscopic

Online-only material: color figures, machine-readable and VO tables

\section{INTRODUCTION}

Transiting exoplanets provide us with the primary source of information about planets outside our own solar system. These are the only planets for which we can routinely and accurately measure both mass and radius. In addition, they provide the possibility for further follow-up observations to measure other physical properties such as brightness, temperature (e.g., Knutson et al. 2007), spin-orbit alignment (e.g., Queloz et al. 2000a), and atmospheric composition (e.g., Charbonneau et al. 2002).

There are 187 transiting exoplanets with published masses and radii, ${ }^{12}$ primarily discovered by the dedicated transit surveys of WASP (Pollacco et al. 2006), HATNet (Bakos et al. 2004), COROT (Auvergne et al. 2009), and Kepler (Borucki et al. 2010). A class of planets known as "hot Jupiters," with short periods $(P<10$ days $)$ and masses/radii similar to Jupiter, account for the majority of these discoveries. Hot Jupiters appear to be rare, occurring at a rate of around $0.4 \%$ around solar-type stars as determined by transit surveys (Bayliss \& Sackett 2011; Fressin et al. 2013). This rarity, coupled with the difficulty in detecting

\footnotetext{
* The HATSouth network is operated by a collaboration consisting of Princeton University (PU), the Max Planck Institute für Astronomie (MPIA), and the Australian National University (ANU). The station at Las Campanas Observatory ( $\mathrm{LCO}$ ) of the Carnegie Institute is operated by PU in conjunction with collaborators at the Pontificia Universidad Católica de Chile (PUC), the station at the High Energy Spectroscopic Survey (HESS) site is operated in conjunction with MPIA, and the station at Siding Spring Observatory (SSO) is operated jointly with ANU.

${ }^{10}$ Alfred P. Sloan Research Fellow.

11 Packard Fellow.

$12 \mathrm{http} / / /$ exoplanets.org, as of 2013 June 1.
}

the $\sim 1 \%$ transit feature from a typical hot Jupiter, has meant that the task of building up a statistically significant set of hot Jupiters has progressed relatively slowly. However, the task is important for two primary reasons. First, individual systems can be studied in great detail to probe the nature of the exoplanet. Second, global trends for giant planets that require a statistically significant sample can be uncovered to better understand the formation and migration of planets.

The discovery of HATS-3b fits into both of these categories. With a host star magnitude of $V=12.4$, it is a promising system for future spectroscopic and photometric follow-up studies, while it adds to the small set of known planets for which orbital and physical properties have been precisely measured.

The layout of the paper is as follows. In Section 2, we report the detection of the photometric signal and the followup spectroscopic and photometric observations of HATS-3. In Section 3, we describe the analysis of the data, beginning with the determination of the stellar parameters, continuing with a discussion of the methods used to rule out non-planetary, false-positive scenarios that could mimic the photometric and spectroscopic observations, and finishing with a description of our global modeling of the photometry and radial velocities (RVs). The discovery of HATS-3b is discussed in Section 4, along with how it fits into the landscape of known hot Jupiters.

\section{OBSERVATIONS}

\subsection{Photometric Detection}

The exoplanet HATS-3b was first identified as a transiting exoplanet candidate based on 14,719 photometric observations 
Table 1

Summary of Photometric Observations

\begin{tabular}{|c|c|c|c|c|}
\hline Facility & Date(s) & Number of Images ${ }^{a}$ & Cadence $(s)^{b}$ & Filter \\
\hline \multicolumn{5}{|l|}{ Discovery } \\
\hline HS-2 (Chile) & 2009 Sep-2010 Sep & 5660 & 284 & Sloan $r$ \\
\hline HS-4 (Namibia) & 2009 Sep-2010 Sep & 8860 & 288 & Sloan $r$ \\
\hline HS-6 (Australia) & 2010 Aug-2010 Sep & 198 & 288 & Sloan $r$ \\
\hline \multicolumn{5}{|l|}{ Follow-up } \\
\hline FTS/Spectral & 2012 Jun 20 & 143 & 50 & Sloan $i$ \\
\hline FTS/Spectral & 2012 Jul 15 & 377 & 50 & Sloan $i$ \\
\hline MPG/ESO2.2/GROND & 2012 Aug 21 & 186 & 129 & Sloan $g$ \\
\hline MPG/ESO2.2/GROND & 2012 Aug 21 & 186 & 129 & Sloan $r$ \\
\hline MPG/ESO2.2/GROND & 2012 Aug 21 & 185 & 129 & Sloan $i$ \\
\hline MPG/ESO2.2/GROND & 2012 Aug 21 & 185 & 129 & Sloan $z$ \\
\hline
\end{tabular}

Notes.

${ }^{a}$ Excludes images that were rejected as significant outliers in the fitting procedure.

$\mathrm{b}$ The mode time difference between consecutive points in each light curve. Due to visibility, weather, pauses for focusing, etc., none of the light curves have perfectly uniform time sampling.

of its host star HATS-3 (also known as 2MASS 204949782425436; $\alpha=20^{\mathrm{h}} 49^{\mathrm{m}} 49^{\mathrm{s}} .80, \delta=-24^{\circ} 25^{\prime} 43^{\prime \prime} .7 ; \mathrm{J} 2000$ ), from the HATSouth global network of automated telescopes (Bakos et al. 2013). The first three entries of Table 1 summarize these HATSouth discovery observations. For this particular candidate, observations were primarily performed by the HS2 and HS4 units (in Chile and Namibia, respectively) over the period of a year from 2009 September to 2010 September. The HS6 unit (in Australia) only contributed a small number of images as it was under construction and commissioning during the period when the field containing HATS-3 was most intensively monitored by the HATSouth network.

Details relating to the observation, reduction, and analysis of the HATSouth photometric discovery data are fully described in Bakos et al. (2013). Here, we provide a brief summary of the salient points.

The HATSouth observations consist of four-minute $r$-band exposures produced using 24 Takahashi E180 astrographs (18 cm diameter primary mirrors) coupled to Apogee $4 \mathrm{~K} \times$ 4K U16M Alta CCDs. Photometry is performed using an aperture photometry pipeline, and light curves are detrended using External Parameter Decorrelation (EPD; Bakos et al. 2010) and the Trend Filtering Algorithm (TFA) of Kovács et al. (2005). Light curves are searched for transit events using an implementation of the Box-fitting Least Squares algorithm (Kovács et al. 2002).

We detected a significant transit signal in the light curve of HATS-3 (see Figure 1). Based on this detection we initiated the follow-up procedure detailed below.

\subsection{Reconnaissance Spectroscopy}

The HATSouth global network of telescopes produces well over 100 candidates each year. To efficiently follow up these candidates, we undertake a series of reconnaissance spectroscopic observations before attempting high-resolution spectroscopy. These reconnaissance observations consist of spectral typing candidates (Section 2.2.1) and medium-resolution RVs (Section 2.2.2). The concept of using low-resolution spectra to vet possible exoplanet host stars has been used successfully for RV surveys (e.g., Robinson et al. 2007). However, for transiting exoplanet surveys, systematic spectroscopic follow-up has traditionally only been carried out using high-resolution echelle spectrographs. We therefore provide a detailed account of our low-resolution spectral typing in this section to highlight the advantages it brings to a large transiting exoplanet survey.

\subsubsection{Reconnaissance Spectral Typing}

The aim of spectral classification during reconnaissance spectroscopy is to (1) efficiently identify and reject candidate host stars that are giants and therefore inconsistent (in our photometric regime) with the planet-star scenario, and (2) determine stellar parameters so that we can identify interesting transiting systems and prioritize the follow-up observations. For this purpose, a low-resolution spectrum is typically the first step in following up a HATSouth candidate.

A single spectrum of HATS-3 was obtained using the Wide Field Spectrograph (WiFeS; Dopita et al. 2007) on the Australian National University (ANU) $2.3 \mathrm{~m}$ telescope on 2012 April 10. WiFeS is a dual-arm image slicer integral field spectrograph. For spectral typing we use the blue arm with the B3000 grating, which delivers a resolution of $R=\lambda / \Delta \lambda=3000$ from 3500 to $6000 \AA$ A. Flux calibrations are performed according to Bessell (1999) using spectrophotometric standard stars from Hamuy et al. (1992) and Bessell (1999). Wavelength calibration is performed using exposures of a Ne-Fe-Ar emission lamp. We sum the four brightest image slices delivered by WiFeS, each $1^{\prime \prime}$ in width. A full description of the instrument configurations can be found in Penev et al. (2013).

The stellar properties $T_{\text {eff }}, \log g,[\mathrm{Fe} / \mathrm{H}]$, and interstellar extinction $(E(B-V))$ are derived via a grid search, minimizing the $\chi^{2}$ between the observed spectrum and synthetic templates from the MARCS model atmospheres (Gustafsson et al. 2008). The search intervals are $250 \mathrm{~K}$ in $T_{\text {eff }}$ and $0.5 \mathrm{dex}$ in $\log g$ and $[\mathrm{Fe} / \mathrm{H}]$. A restricted $T_{\text {eff }}$ search space is established using Two Micron All Sky Survey (2MASS) $J-K$ colors. Extinction is applied according to Cardelli et al. (1989), with $E(B-V)$ values ranging from 0 to the maximum extinction from the Schlegel et al. (1998) maps. The $T_{\text {eff }}-\log g$ probability space for HATS-3 is plotted in Figure 2, along with the observed spectrum and best-fitting template.

Since the differentiation of giants and dwarfs is of particular importance, we place more weight on the $\log g$ sensitive spectral features during the $\chi^{2}$ calculations. These regions include the $\mathrm{MgH}$ feature (e.g., Bell et al. 1985; Berdyugina \& Savanov 1994), the Mg $b$ triplet (e.g., Ibata \& Irwin 1997) for cooler stars, and the Balmer jump for hotter stars (e.g., Bessell 2007). 

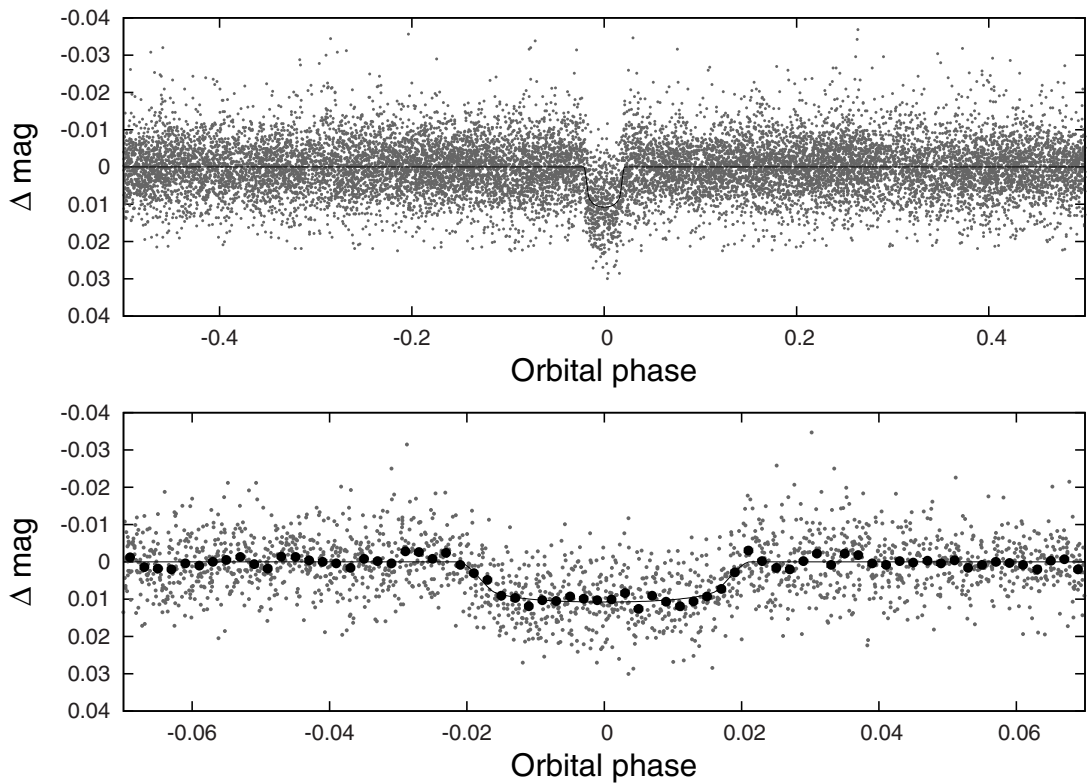

Figure 1. Discovery light curve for HATS-3b, consisting of 14,719 photometric observations from the HATSouth global telescope network. The top panel is the unbinned, relative, instrumental $r$-band light curve, phase-folded to the period $P=3.5478513$ days resulting from the global fit described in Section 3. The lower panel shows a zoom-in around the transit; dark filled circles are the light curve binned at 0.002 in phase. The solid line in both panels is the best-fit transit model (see Section 3). As a result of blending and our noise-filtering procedure, the transit detected in the HATSouth light curve for HATS-3 is 15\% shallower than the true $r$-band transit (see notes in Table 5).
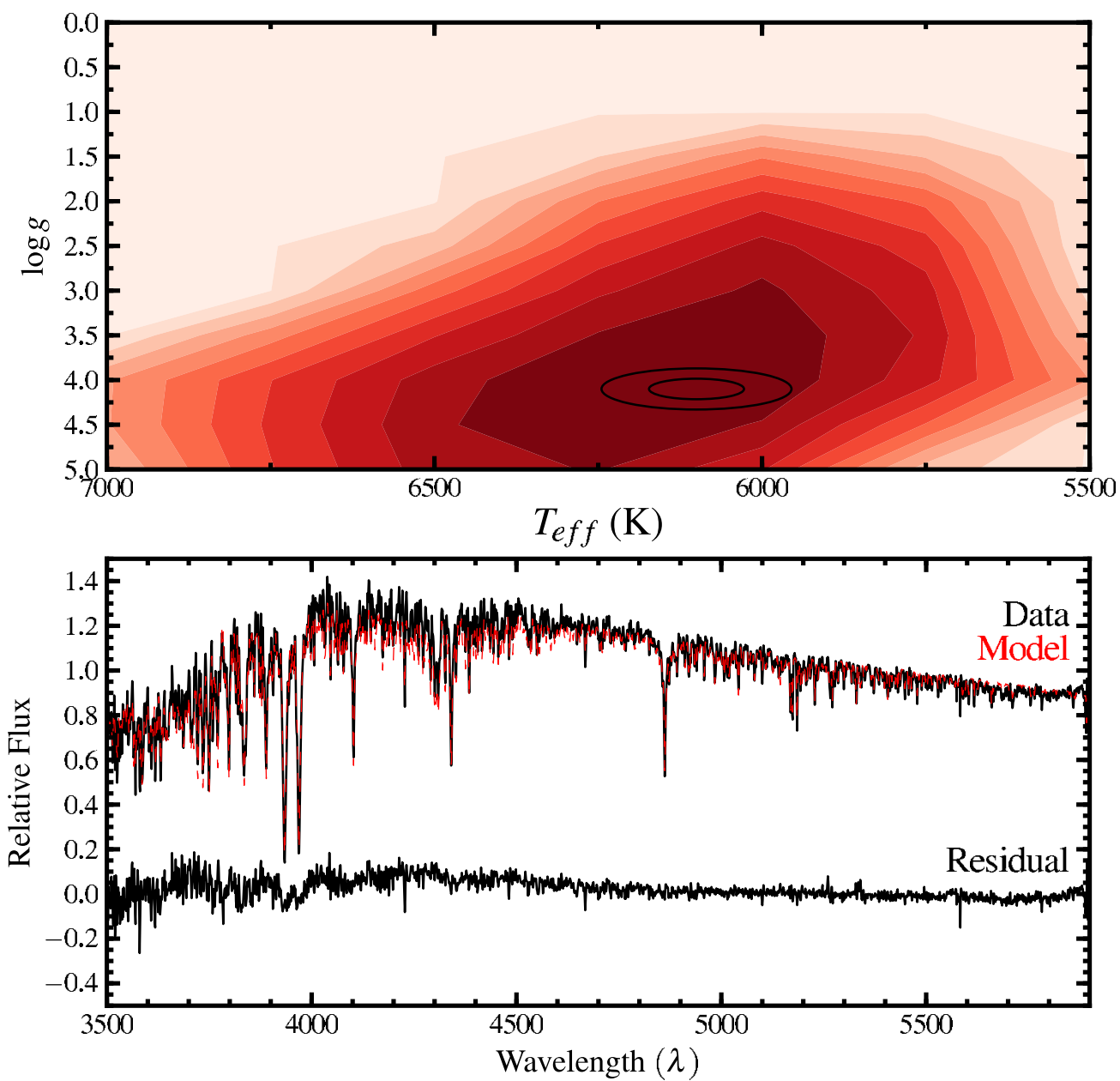

Figure 2. Low-resolution spectral classification of HATS-3 using WiFeS as detailed in Section 2.2.1. Top: contours marking the $T_{\text {eff }}-\log g$ probability space are shown. Typical $1 \sigma$ and $2 \sigma$ uncertainties for WiFeS-derived stellar parameters are shown. Bottom: the observed spectrum (black solid) is plotted against the best-fitting template spectrum (red dashed), in units of relative flux. Residuals are plotted beneath.

(A color version of this figure is available in the online journal.) 
Table 2

WiFeS Reconnaissance Spectral Typing Performance

\begin{tabular}{lllr}
\hline \hline Star (Reference) & \multicolumn{1}{c}{$T_{\mathrm{eff}^{\mathrm{a}}}$} & \multicolumn{1}{c}{$\log g^{\mathrm{a}}$} & {$[\mathrm{Fe} / \mathrm{H}]^{\mathrm{a}}$} \\
\hline WASP-4 (Wilson et al. 2008) & $5500(5500 \pm 150)$ & $4.4(4.45+0.016 /-0.029)$ & $0.0(0.00 \pm 0.20)$ \\
WASP-5 (Anderson et al. 2008) & $5900(5880 \pm 150)$ & $3.9(4.40+0.039 /-0.048)$ & $0.5(0.09 \pm 0.09)$ \\
WASP-7 (Hellier et al. 2009) & $6400(6400 \pm 100)$ & $4.4(4.36+0.01 /-0.047)$ & $-0.5(0.00 \pm 0.1)$ \\
WASP-8 (Queloz et al. 2010) & $5700(5600 \pm 80)$ & $3.7(4.50 \pm 0.1)$ & $-0.5(0.17 \pm 0.07)$ \\
WASP-29 (Hellier et al. 2010) & $4600(4800 \pm 150)$ & $4.4(4.50 \pm 0.2)$ & $0.0(0.11 \pm 0.14)$ \\
WASP-46 (Anderson et al. 2012) & $5900(5620 \pm 160)$ & $4.5(4.49 \pm 0.02)$ & $0.0(-0.37 \pm 0.13)$ \\
HATS-1 (Penev et al. 2013) & $6000(5870 \pm 100)$ & $4.8(4.40 \pm 0.08)$ & $-0.5(-0.06 \pm 0.12)$ \\
HATS-2 (Mohler-Fischer et al. 2013) & $4800(5227 \pm 95)$ & $4.4(4.44 \pm 0.12)$ & $-0.5(0.15 \pm 0.05)$ \\
HATS-3 (This work.) & $6200(6351 \pm 76)$ & $4.1(4.23 \pm 0.0)$ & $-0.5(-0.157 \pm 0.07)$ \\
HD36702 (de Medeiros et al. 2006) & $4800(4485 \pm 111)$ & $0.2(0.8 \pm 0.15)$ & $-2.0(-2.0 \pm 0.17)$ \\
HD29574 (de Medeiros et al. 2006) & $4500(4310 \pm 111)$ & $0.3(0.6 \pm 0.15)$ & $-2.0(-1.9 \pm 0.17)$ \\
HD26297 (de Medeiros et al. 2006) & $4800(4500 \pm 111)$ & $0.3(1.2 \pm 0.15)$ & $-1.5(-1.7 \pm 0.17)$ \\
HD20453 (de Medeiros et al. 2006) & $5100(5365 \pm 111)$ & $0.9(1.2 \pm 0.15)$ & $-1.5(-2.0 \pm 0.17)$ \\
HD103036 (de Medeiros et al. 2006) & $4700(4375 \pm 111)$ & $0.3(0.8 \pm 0.15)$ & $-1.5(-1.7 \pm 0.17)$ \\
HD122956 (de Medeiros et al. 2006) & $4800(4575 \pm 111)$ & $0.9(1.1 \pm 0.15)$ & $-2.0(-1.8 \pm 0.17)$ \\
\hline
\end{tabular}

Note. ${ }^{a}$ Literature value given in parentheses.

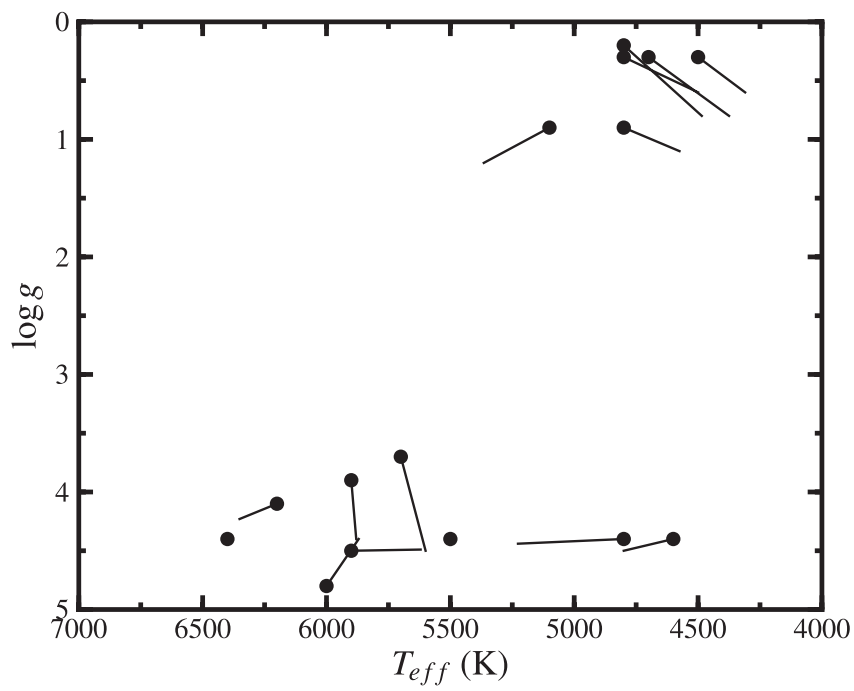

Figure 3. Benchmarking the WiFeS spectral classifications as discussed in Section 2.2.1. $T_{\text {eff } \star}$ and $\log g$ values derived from our WiFeS spectral classification are marked by solid points, the vectors point toward the corresponding published values as set out in Table 2 .

To estimate the uncertainties of the HATSouth reconnaissance spectral typing, we observed nine planet-hosting stars with published stellar parameters derived from high-resolution spectroscopy (including the HATS-3 properties presented in this study). The range in $T_{\mathrm{eff}}, \log g,[\mathrm{Fe} / \mathrm{H}]$, and $V$ magnitude of these stars closely resemble the HATSouth candidates, so they serve as good bench markers. In addition, we also include six evolved stars with published stellar parameters from high-resolution spectroscopy (de Medeiros et al. 2006). These stars have low $\log g$ values and demonstrate our ability to distinguish between giants and dwarfs. The results are presented in Table 2 and Figure 3 . The rms deviation between the parameters derived from our WiFeS observations of the dwarf stars and their published parameters are $T_{\mathrm{eff}_{\star}}=200 \mathrm{~K}, \log g=0.35$, and $[\mathrm{Fe} / \mathrm{H}]=0.44$.

WiFeS low-resolution reconnaissance spectral classification revealed that HATS-3 is an F dwarf with $T_{\mathrm{eff} \star}=6200 \pm 200 \mathrm{~K}$, $\log g_{\star}=4.1 \pm 0.4$, and $[\mathrm{Fe} / \mathrm{H}]=-0.5 \pm 0.4$. These values are consistent with the more precise values obtained from subsequent high-resolution spectroscopy (Section 2.3).
To date, $12 \%$ of the 240 HATSouth candidates spectral typed by our reconnaissance spectroscopy are giants $(\log g<$ 3.5). For the expected galactic population we are monitoring, approximated using the Besançon model (Robin et al. 2003), within a field centered at $\alpha=300^{\circ}, \delta=25^{\circ}$ and extending to $50 \mathrm{kpc}$ distance, the giant occurrence rate is $24 \%$. The fraction of our candidates that are giants is much greater for brighter candidates, as is quantified in Figure 4. This is expected, as the overall giant fraction is higher for brighter magnitude ranges (see Figure 4). We find that $35 \%$ of HATSouth candidates with magnitudes $9<V<12$ are giants, making reconnaissance spectral typing especially valuable over this magnitude range.

\subsubsection{Reconnaissance WiFeS Radial Velocities}

In addition to determining stellar parameters, we also use WiFeS on the ANU $2.3 \mathrm{~m}$ telescope to look for RV variations above $\sim 2 \mathrm{~km} \mathrm{~s}^{-1}$. Such variations indicate that the transiting body is typically a stellar-mass object rather than an exoplanet, and effectively rules out the candidate as a transiting exoplanet. The observations are timed to phase quadratures, where the expected velocity difference is greatest.

For RV measurements, we use the red arm of the WiFeS spectrograph with the R7000 grating and RT480 dichroic. This results in $R \equiv \lambda / \Delta \lambda=7000$ over 5200-7000 $\AA$. Wavelength solutions are provided by bracketing $\mathrm{Ne}-\mathrm{Fe}-\mathrm{Ar}$ arc lamp exposures, with a further first-order correction made using telluric oxygen $B$-band lines at 6882-6906 $\AA$. RVs are derived via cross-correlation against RV standard star (Nidever et al. 2002) exposures taken every night. Further details of the observing setup and the data reduction pipeline can be found in Penev et al. (2013).

Simultaneous radial velocities are also derived for any neighbors within the $12 \times 38^{\prime \prime} \mathrm{WiFeS}$ field of view. Significant velocity variations for any close-neighbors consistent with the photometric ephemeris are indicative of blended eclipsing binary scenarios, and are subsequently rejected.

To date, 184 HATSouth candidates have been monitored using WiFeS multi-epoch RV measurements with enough phase coverage to constrain any velocity variation at the $2 \mathrm{~km} \mathrm{~s}^{-1}$ level. We find 51 of our candidates to be stellar mass binaries, which equates to a $27 \%$ contamination rate. Figure 5 presents the distribution of the RV orbit semi-amplitude $(K)$ of these 


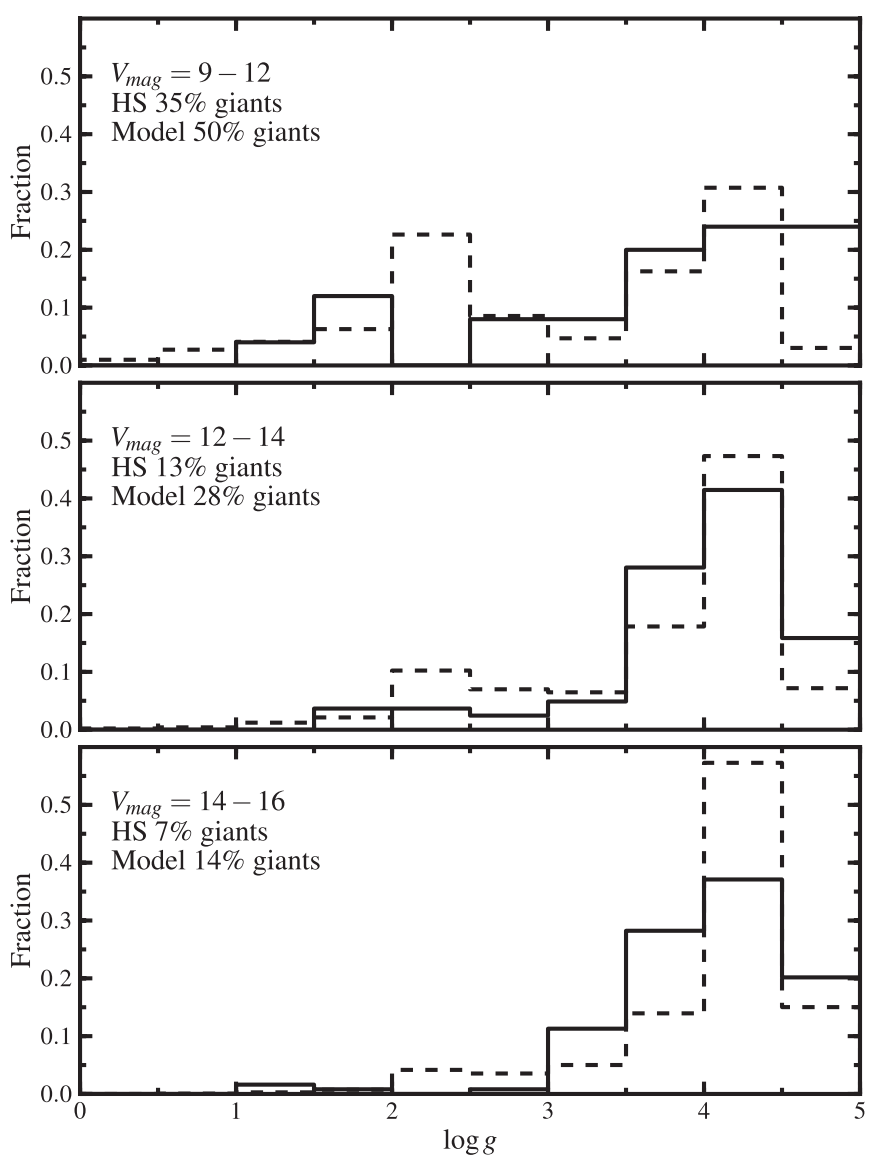

Figure 4. Histograms showing the $\log g$ distribution for HATSouth candidates over different brightness bins based on the WiFeS spectral classification set out in Section 2.2.1. The candidate population is plotted by solid lines and the Besançon model population (Robin et al. 2003) is plotted in dashed lines for reference. The contribution by giants is noted in each brightness bin.

candidates. We find two peaks in the velocity amplitude, one at $\sim 10 \mathrm{~km} \mathrm{~s}^{-1}$, representing the $\mathrm{F}-\mathrm{M}$ stellar binary population, and another at $\sim 45 \mathrm{~km} \mathrm{~s}^{-1}$, representing a larger mass ratio population that exhibits shallow grazing transits. In both scenarios, the resulting transit depths are similar to that of a planet-star system, and are identified as potential planetary systems from their discovery light curve.

As in Section 2.2.1 we find that the fraction of these contaminating systems varies strongly with candidate magnitude; however, this time it is the fainter candidates that are more heavily contaminated; see Figure 5. We find a paucity of eclipsing binaries for the brighter candidates. The reason for this is that these light curves are of higher precision that allows better determination of the shape of the transit feature. Also, as was pointed out in Section 2.2.1, a higher fraction of the brighter candidates are giants. The large number of contaminating eclipsing binaries for the faintest candidates highlights the particular advantage of medium-resolution RV observations in this regime. Although faint, these candidates are of interest to the HATSouth survey as they contain a high fraction of low-mass stars that are not easily probed by other wide-field transit surveys.

We obtained one $500 \mathrm{~s}$ exposure on each of three consecutive nights over 2012 April 10-12 to measure the RV for HATS-3 over its full phase. The RVs were clustered within $1 \mathrm{~km} \mathrm{~s}^{-1}$ of each other on each night, indicating that the transiting body could not be of stellar mass.
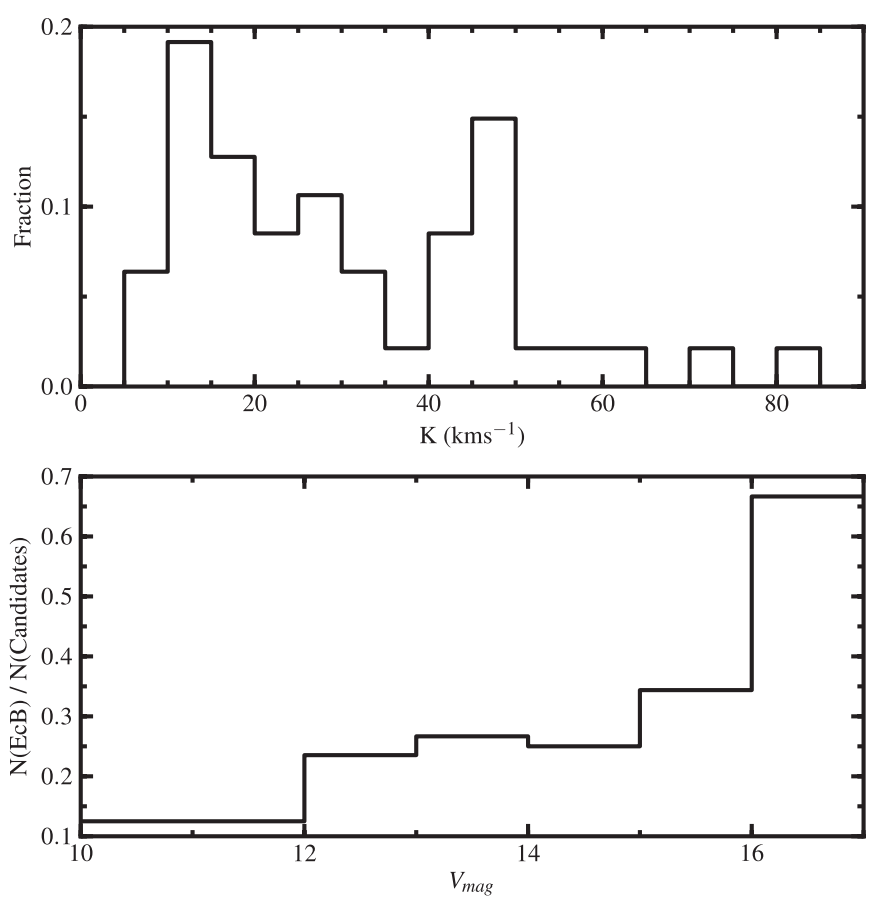

Figure 5. Top: distribution of radial velocity semi-amplitude $(K)$ for the stellar mass binaries identified from WiFeS medium-resolution measurements (Section 2.2.2). Bottom: fractional contamination by eclipsing binaries in the HATSouth candidate population as a function of $V$-band magnitude.

\subsection{High-resolution Spectroscopy}

High-resolution spectroscopy is only carried out on candidates that pass the screening process involved with the reconnaissance spectroscopy set out in Section 2.2. This allows us to focus our time-intensive high-resolution spectroscopy on the targets that are most likely to host planets.

HATS-3 was monitored by three different echelle spectrographs capable of measuring high-precision RVs over the period 2012 April-June. The high $v \sin i$ and $T_{\text {eff } \star}$ of HATS-3 (see Section 3.1) meant obtaining precise RVs was a challenge, as the stellar absorption lines used for cross-correlation were fewer and more highly broadened than a solar-type star. Table 3 summarizes these high-resolution spectroscopic observations. Thirteen observations were taken with FEROS (Kaufer \& Pasquini 1998) on the Max Planck Gesellschaft (MPG) ESO $2.2 \mathrm{~m}$ telescope at La Silla Observatory, Chile. A further eight observations of HATS-3 were taken by both CORALIE (Queloz et al. 2000b) on the Swiss Leonard Euler $1 \mathrm{~m}$ telescope at La Silla Observatory, Chile, and UCLES (using the CYCLOPS fiber feed) on the $3.9 \mathrm{~m}$ Anglo-Australian Telescope (AAT) at Siding Spring Observatory, Australia. For a description of the observations and data reduction employed for these instruments, see Penev et al. (2013). The RV measurements for these observations are set out in Table 4, and are plotted after phase-wrapping to the best-fit period (see Section 3) in Figure 6. We calculated the bisector spans of the cross-correlation functions (CCFs) by determining the mid-points of the CCFs between 0.4 and 0.8 of the full peak height, and then fitting a straight line to these midpoints. The bisectors are taken as the inverse of the gradients of these lines. We find the CCF bisectors do not vary in phase with the RV measurements.

\subsection{Photometric Follow-up Observations}

High-precision photometric follow-up is important in determining the precise orbital parameters of the exoplanet system 
Table 3

Summary of Spectroscopic Observations

\begin{tabular}{lcccrrr}
\hline \hline $\begin{array}{l}\text { Telescope/ } \\
\text { Instrument }\end{array}$ & \multicolumn{1}{c}{$\begin{array}{c}\text { Date } \\
\text { Range }\end{array}$} & $\begin{array}{c}\text { Number of } \\
\text { Observations }\end{array}$ & $\begin{array}{c}\text { Exposure } \\
\text { Times (s) }\end{array}$ & Resolution & S/N $\mathrm{N}^{\mathrm{a}}$ & $\begin{array}{c}\text { Wavelength } \\
\text { Coverage }(\AA)\end{array}$ \\
\hline $\begin{array}{l}\text { Reconnaissance } \\
\text { ANU 2.3 m/WiFeS }\end{array}$ & 2012 Apr 10 & 1 & 300 & 3000 & 100 & $3500-6000$ \\
ANU 2.3 m/WiFeS & 2012 Apr 10-12 & 3 & 500 & 7000 & 50 & $5200-7000$ \\
High-precision radial velocity & & & & & & \\
AAT 3.9 m/CYCLOPS & 2012 May 5-11 & 8 & 1500 & 70,000 & 20 & $4540-7340$ \\
Euler 1.2 m/Coralie & 2012 Jun 2-7 & 8 & 1800 & 60,000 & 20 & $3850-6900$ \\
MPG/ESO 2.2 m/FEROS & 2012 Apr 1-Jun 8 & 13 & 2700 & 48,000 & 20 & $3500-9200$ \\
\hline
\end{tabular}

Note. ${ }^{\text {a }}$ The approximate signal-to-noise ratio $(\mathrm{S} / \mathrm{N})$ per resolution element.

Table 4

Relative Radial Velocity Measurements of HATS-3

\begin{tabular}{|c|c|c|c|c|}
\hline $\begin{array}{l}\text { BJD } \\
(2,454,000+)\end{array}$ & $\begin{array}{c}\mathrm{RV}^{\mathrm{a}} \\
\left(\mathrm{m} \mathrm{s}^{-1}\right)\end{array}$ & $\begin{array}{c}\sigma_{\mathrm{RV}}^{\mathrm{b}} \\
\left(\mathrm{m} \mathrm{s}^{-1}\right)\end{array}$ & Phase & Instrumen \\
\hline 2019.89700 & 124 & 53 & 0.647 & FEROS \\
\hline 2032.87600 & 36 & 108 & 0.305 & FEROS \\
\hline 2035.90600 & -77 & 45 & 0.159 & FEROS \\
\hline 2036.88374 & 115 & 28 & 0.435 & Coralie \\
\hline 2037.92527 & 155 & 33 & 0.729 & Coralie \\
\hline 2040.85700 & 108 & 89 & 0.555 & FEROS \\
\hline 2053.19936 & -116 & 45 & 0.034 & AAT \\
\hline 2053.32636 & -180 & 94 & 0.070 & AAT \\
\hline 2055.25949 & 4 & 27 & 0.614 & AAT \\
\hline 2056.20988 & 161 & 29 & 0.882 & AAT \\
\hline 2057.17510 & -159 & 34 & 0.154 & AAT \\
\hline 2057.32183 & -20 & 29 & 0.196 & AAT \\
\hline 2058.24637 & -32 & 25 & 0.456 & AAT \\
\hline 2059.25685 & 210 & 34 & 0.741 & AAT \\
\hline 2076.83800 & 173 & 65 & 0.697 & FEROS \\
\hline 2077.74700 & 84 & 99 & 0.953 & FEROS \\
\hline 2078.76300 & -163 & 64 & 0.239 & FEROS \\
\hline 2079.79400 & 54 & 58 & 0.530 & FEROS \\
\hline 2080.75400 & 149 & 61 & 0.800 & FEROS \\
\hline 2080.83500 & -8 & 46 & 0.823 & FEROS \\
\hline 2080.83735 & 64 & 20 & 0.824 & Coralie \\
\hline 2081.73600 & -154 & 63 & 0.077 & FEROS \\
\hline 2081.91580 & -163 & 21 & 0.128 & Coralie \\
\hline 2082.90893 & -56 & 25 & 0.408 & Coralie \\
\hline 2083.80200 & 135 & 109 & 0.659 & FEROS \\
\hline 2083.83791 & 139 & 21 & 0.670 & Coralie \\
\hline 2084.93164 & -38 & 22 & 0.978 & Coralie \\
\hline 2085.91066 & -162 & 21 & 0.254 & Coralie \\
\hline 2086.84600 & 35 & 53 & 0.517 & FEROS \\
\hline
\end{tabular}

Notes.

a The zero-point of these velocities is arbitrary. An overall offset $\gamma_{\text {rel }}$ fitted separately to the CORALIE, FEROS, and CYCLOPS velocities in Section 3 has been subtracted.

b Internal errors excluding the component of astrophysical/instrumental jitter considered in Section 3.

and the planetary radius. We used two facilities for this task: the Spectral camera on the $2.0 \mathrm{~m}$ Faulkes Telescope South (FTS) and the GROND camera on the MPG/ESO $2.2 \mathrm{~m}$ telescope. The high-precision photometric follow-up data are presented in Table 5, and a summary is presented in Table 1.

\subsubsection{FTS $2 \mathrm{~m} /$ Spectral}

FTS is a fully automated, robotic telescope operated as part of the Las Cumbres Observatory Global Telescope (LCOGT) Network. The queue-based scheduling allows for transiting

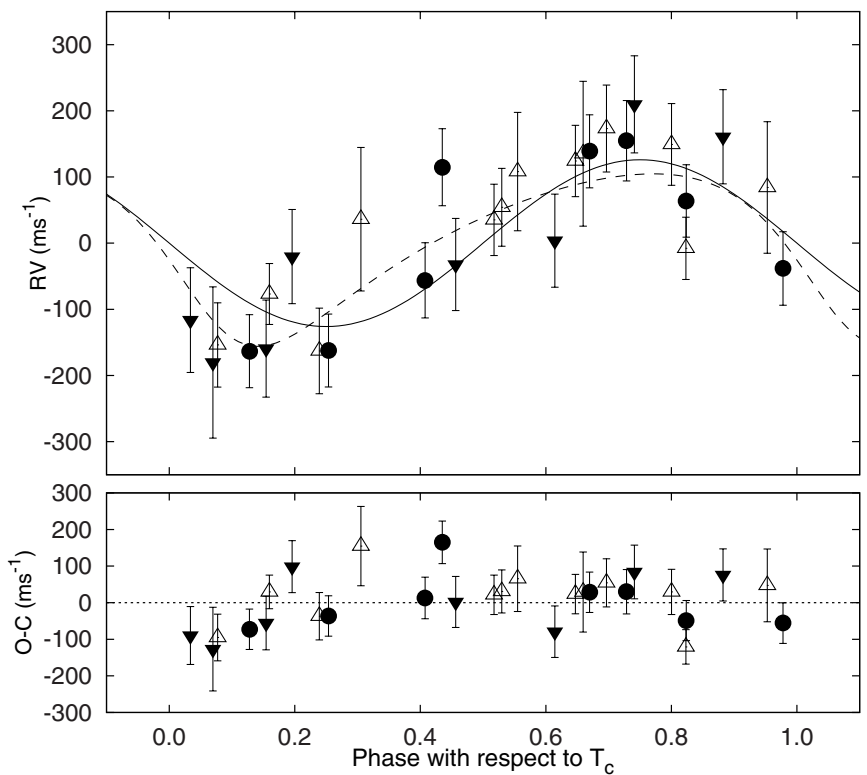

Figure 6. Top panel: radial velocity measurements, as detailed in Section 2.3, for HATS-3 from CORALIE (dark filled circles), FEROS (open triangles), and CYCLOPS (filled triangles) shown as a function of orbital phase, together with our best-fit circular (solid line) and eccentric (dotted line) models, as discussed in Section 3.3. Zero phase corresponds to the time of mid-transit. The centerof-mass velocity has been subtracted. Second panel: velocity $O-C$ residuals from the best-fit circular model. The error bars include a component from astrophysical/instrumental jitter allowed to differ for the three instruments.

planets to be easily monitored at the time of the transit event. The "spectral" camera with an $i$-band filter is employed for our transit observations of HATS-3b. The camera has a $4 \mathrm{~K} \times 4 \mathrm{~K}$ array of 0 '. 15 pixels, and we use it with $2 \times 2$ binning to reduce readout time. The telescope is slightly defocused to reduce the effect of imperfect flat-fielding and to allow for slightly longer exposure times without saturating. For HATS-3 the exposures were $30 \mathrm{~s}$, which provided for $50 \mathrm{~s}$ cadence photometry given the CCD readout time of the spectral camera. The raw fits files are reduced automatically via the LCOGT reduction pipeline, which includes flat-field correction and fitting of an astrometric solution. Photometry is performed on the reduced images using an automated pipeline based on Source Extractor (version 2.5; Bertin \& Arnouts 1996), which calculates the photometry in fixed circular apertures after subtracting a background map made using the entire image.

On the night of 2012 June 20, we monitored HATS-3 with FTS. Although the observations only started mid-ingress and finished before the egress began, this information allowed us to update the period and phase, and alert us to the need for further 
Table 5

Differential Photometry of HATS-3

\begin{tabular}{lrcccc}
\hline \hline $\begin{array}{l}\text { BJD } \\
(2,400,000+)\end{array}$ & Mag $^{\mathrm{a}}$ & $\sigma_{\mathrm{Mag}}$ & Mag(orig) $^{\mathrm{b}}$ & Filter & Instrument \\
\hline 55100.48159 & -0.00404 & 0.00277 & $\ldots$ & $r$ & $\mathrm{HS}$ \\
55366.57071 & 0.00091 & 0.00242 & $\ldots$ & $r$ & $\mathrm{HS}$ \\
55334.64007 & 0.01110 & 0.00268 & $\ldots$ & $r$ & $\mathrm{HS}$ \\
55359.47536 & 0.00753 & 0.00245 & $\ldots$ & $r$ & $\mathrm{HS}$ \\
55391.40621 & -0.00212 & 0.00256 & $\ldots$ & $r$ & $\mathrm{HS}$ \\
55380.76328 & -0.00029 & 0.00250 & $\ldots$ & $r$ & HS \\
55437.52939 & -0.00387 & 0.00252 & $\ldots$ & $r$ & HS \\
55423.33825 & 0.00704 & 0.00250 & $\ldots$ & $r$ & HS \\
55437.52985 & 0.00690 & 0.00425 & $\ldots$ & $r$ & HS \\
55093.38841 & 0.00932 & 0.00260 & $\ldots$ & $r$ & HS \\
\hline
\end{tabular}

Notes.

a The out-of-transit level has been subtracted. For the HATSouth light curve (rows with "HS" in the Instrument column), these magnitudes have been detrended using the EPD and TFA procedures prior to fitting a transit model to the light curve. Primarily as a result of this detrending, but also due to blending from neighbors, the apparent HATSouth transit depth is $\sim 86 \%$ that of the true depth in the Sloan $r$ filter. For the follow-up light curves (rows with an Instrument other than "HS") these magnitudes have been detrended with the EPD and TFA procedures, carried out simultaneously with the transit fit (the transit shape is preserved in this process)

b Raw magnitude values without application of the EPD and TFA procedures. This is only reported for the follow-up light curves.

(This table is available in its entirety in machine-readable and Virtual Observatory (VO) forms in the online journal. A portion is shown here for guidance regarding its form and content.)

follow-up photometry. On 2012 July 15 we again monitored HATS-3 with FTS, this time covering the entire transit event. This light curve is presented in Figure 7.

\subsubsection{ESO $2.2 \mathrm{~m} / \mathrm{GROND}$}

GROND is a seven-channel imager on the MPG/ESO $2.2 \mathrm{~m}$ telescope at La Silla Observatory in Chile (Greiner et al. 2008). Though it is primarily designed for rapid observations of gamma-ray burst afterglows, it has proved a very useful instrument for multi-band, high-precision follow-up light curves for transiting planets in general (e.g., Mancini et al. 2013), and in particular HATSouth planet discoveries (e.g., Penev et al. 2013; Mohler-Fischer et al. 2013).

On 2012 August 26 we used GROND to simultaneously monitor an entire transit of HATS-3b in four bands, similar to Sloan $g, r, i$, and $z$ bands). The telescope was defocused, and the exposure time was set to $62 \mathrm{~s}$ in all bands, which resulted in an effective cadence of $129 \mathrm{~s}$. The data were reduced in the standard manner, and aperture photometry was performed using an IDL/ATROLIB implementation of DAOPHOT (Stetson 1987; Southworth et al. 2009). The light curves for each of the four bands are set out in Figure 7.

\section{ANALYSIS}

\subsection{Properties of the Host Star HATS-3}

The stellar parameters of HATS-3, including $T_{\text {eff }}, \log g_{\star}$, $[\mathrm{Fe} / \mathrm{H}]$, and $v \sin i$, are derived from the high-resolution FEROS spectra. In a procedure similar to that presented in MohlerFischer et al. (2013), the 13 spectra were analyzed using the "Spectroscopy Made Easy" software package (SME; Valenti \& Piskunov 1996). The stellar parameters we list are the mean of the values derived from each spectrum, weighted by the $\mathrm{S} / \mathrm{N}$

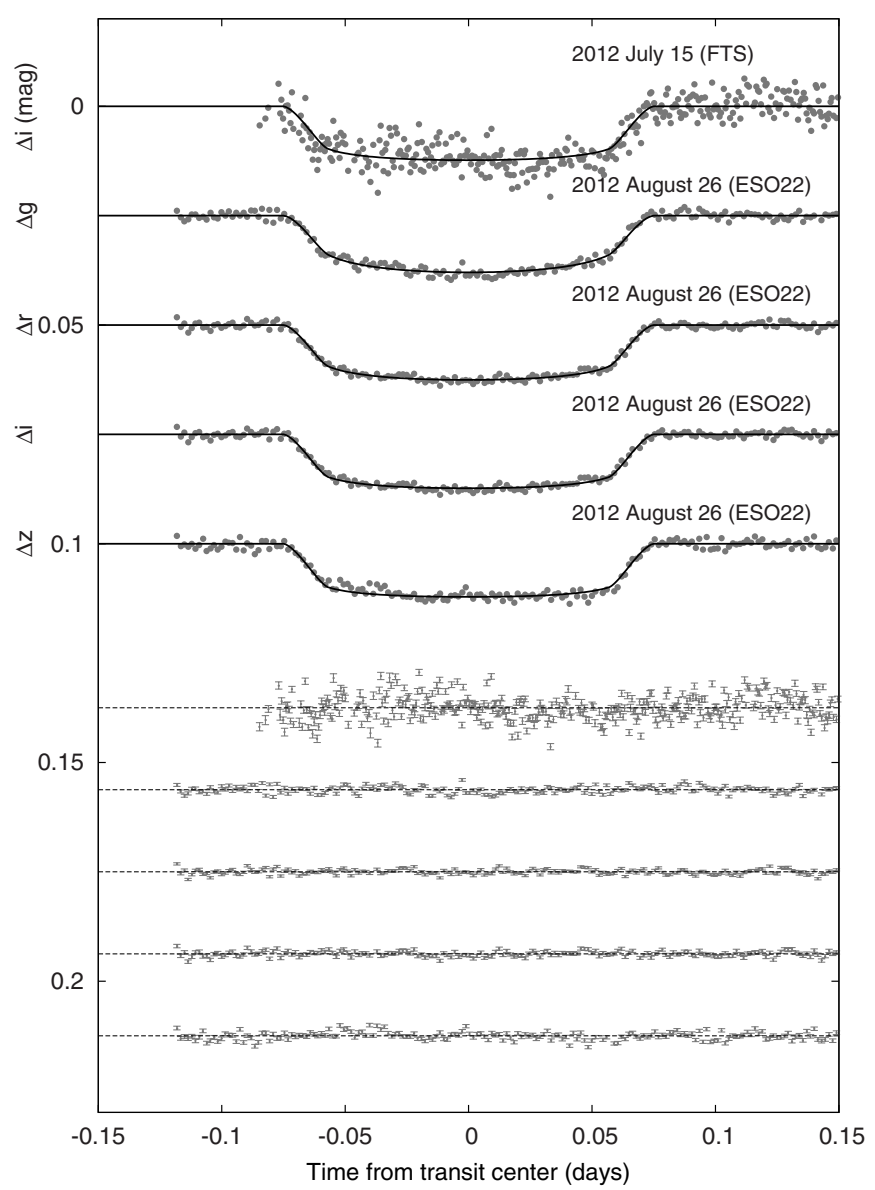

Figure 7. Unbinned instrumental Sloan $g_{-}, r_{-}, i_{-}$, and $z$-band transit light curves of HATS-3, as detailed in Section 2.4. The dates and instruments used for each event are indicated. The light curves have been detrended using the EPD and TFA processes. Light curves are shifted for clarity. Our best fit is shown by the solid lines. Residuals from the fits are displayed at the bottom, in the same order as the top light curves.

of the spectrum. The uncertainties in the stellar parameters are derived from the distribution of these parameters over the 13 spectra.

Following the method described in Sozzetti et al. (2007) and applied in Penev et al. (2013), we determine fundamental stellar properties (mass, radius, age, and luminosity) based on the mean stellar density derived from the light-curve fitting (which is directly related to $\left.a / R_{\star}\right)$, the $T_{\mathrm{eff} \star}$ from the SME analysis, and the Yonsei-Yale (YY; Yi et al. 2001) stellar evolution models. This analysis provides a value of $\log g_{\star}=4.23 \pm 0.01$, which is more precise than can be obtained via SME alone. We then fix this as the $\log g_{\star}$ for HATS-3 and repeat the SME analysis to determine the final stellar parameters listed in Table 6 . The $1 \sigma$ and $2 \sigma$ confidence ellipsoids in $T_{\text {eff } \star}$ and $a / R_{\star}$ are determined from the posterior distribution $a / R_{\star}$ from our Markov Chain Monte Carlo (MCMC) modeling (see Section 3.3) coupled with an assumed Gaussian distribution for the effective temperature as returned from SME. These ellipsoids are plotted in Figure 8, along with the YY isochrones for the SME determined $[\mathrm{Fe} / \mathrm{H}]$, and a range of stellar ages. We find that HATS-3 is an F dwarf host, the first for the HATSouth survey, with $T_{\text {eff } \star}=6351 \pm 76 \mathrm{~K}$.

\subsection{Excluding Blends}

To rule out the possibility that HATS-3 is a blended stellar binary system that mimics the observable properties 
Table 6

Stellar Parameters for HATS-3

\begin{tabular}{|c|c|c|c|}
\hline Parameter & $\begin{array}{c}\text { Value } \\
\text { Circular }\end{array}$ & $\begin{array}{c}\text { Value } \\
\text { Eccentric }\end{array}$ & Source \\
\hline \multicolumn{4}{|c|}{ Spectroscopic properties } \\
\hline$T_{\mathrm{eff} \star}(\mathrm{K})$ & $6351 \pm 76$ & $6325 \pm 83$ & $\mathrm{SME}^{\mathrm{a}}$ \\
\hline$[\mathrm{Fe} / \mathrm{H}]$ & $-0.157 \pm 0.07$ & $-0.139 \pm 0.09$ & SME \\
\hline$v \sin i\left(\mathrm{~km} \mathrm{~s}^{-1}\right)$ & $9.12 \pm 1.31$ & $8.97 \pm 1.8$ & SME \\
\hline \multicolumn{4}{|c|}{ Photometric properties } \\
\hline$V(\operatorname{mag})$ & $12.44 \pm 0.2$ & $12.44 \pm 0.2$ & APASS \\
\hline$B(\mathrm{mag})$ & $12.46 \pm 0.18$ & $12.46 \pm 0.18$ & APASS \\
\hline$J$ (mag) & $10.980 \pm 0.021$ & $10.980 \pm 0.021$ & 2MASS \\
\hline$H(\mathrm{mag})$ & $10.719 \pm 0.024$ & $10.719 \pm 0.024$ & 2MASS \\
\hline$K_{s}(\mathrm{mag})$ & $10.694 \pm 0.021$ & $10.694 \pm 0.021$ & 2MASS \\
\hline \multicolumn{4}{|l|}{ Derived properties } \\
\hline$M_{\star}\left(M_{\odot}\right)$ & $1.209 \pm 0.036$ & $1.300 \pm 0.088$ & $\mathrm{YY}+a / R_{\star}+\mathrm{SME}^{\mathrm{b}}$ \\
\hline$R_{\star}\left(R_{\odot}\right)$ & $1.404 \pm 0.030$ & $1.725 \pm 0.242$ & $\mathrm{YY}+a / R_{\star}+\mathrm{SME}$ \\
\hline $\log g_{\star}(\mathrm{cgs})$ & $4.22 \pm 0.01$ & $4.08 \pm 0.10$ & $\mathrm{YY}+a / R_{\star}+\mathrm{SME}$ \\
\hline$L_{\star}\left(L_{\odot}\right)$ & $2.86 \pm 0.21$ & $4.28_{-1.02}^{+1.53}$ & $\mathrm{YY}+a / R_{\star}+\mathrm{SME}$ \\
\hline$M_{V}(\mathrm{mag})$ & $3.62 \pm 0.09$ & $3.19 \pm 0.31$ & $\mathrm{YY}+a / R_{\star}+\mathrm{SME}$ \\
\hline$M_{K}(\mathrm{mag}, \mathrm{ESO})$ & $2.46 \pm 0.05$ & $2.01 \pm 0.30$ & $\mathrm{YY}+a / R_{\star}+\mathrm{SME}$ \\
\hline Age (Gyr) & $3.2_{-0.4}^{+0.6}$ & $3.1_{-0.4}^{+0.8}$ & $\mathrm{YY}+a / R_{\star}+\mathrm{SME}$ \\
\hline Distance (pc) & $453 \pm 11$ & $556 \pm 78$ & $\mathrm{YY}+a / R_{\star}+\mathrm{SME}$ \\
\hline$E(B-V)$ & $0.098 \pm 0.054$ & $0.096 \pm 0.053$ & $\mathrm{YY}+a / R_{\star}+\mathrm{SME}$ \\
\hline
\end{tabular}

Notes.

" SME: "Spectroscopy Made Easy" package for the analysis of high-resolution spectra (Valenti \& Piskunov 1996). These parameters rely primarily on SME, but have a small dependence also on the iterative analysis incorporating the isochrone search and global modeling of the data, as described in the text.

b $\mathrm{YY}+a / R_{\star}+\mathrm{SME}$ : based on the YY isochrones (Yi et al. 2001), $a / R_{\star}$ as a luminosity indicator, and the SME results (see Section 3.1).

of a transiting planet system, we conducted a blend analysis following the procedure described in Hartman et al. (2011). This procedure involves modeling the photometric light curves, photometry taken from public catalogs and calibrated to an absolute scale, and spectroscopically determined stellar atmospheric parameters. We compare the fits from a model consisting of a single planet transiting a star, to models of blended stellar systems with components having properties constrained by stellar evolution models.

We find that the single-star plus transiting planet model fits the data better than any non-planetary blend scenario. As is typically the case, there are some scenarios in which the two brightest components in the blend are of similar mass that we cannot rule out with greater than $5 \sigma$ confidence based on the photometry and atmospheric parameters. However, in all such cases there would be a secondary component in the blended object with a brightness that is $>38 \%$ that of the primary star. That secondary component would itself be undergoing an orbit with a semiamplitude of several tens of $\mathrm{km} \mathrm{s}^{-1}$. Such a blend would have been easily detected, either as a double-lined spectrum, or via several $\mathrm{km} \mathrm{s}^{-1}$ bisector-span variations.

\subsection{Global Modeling of Data}

In order to determine the physical planetary parameters, we carried out a joint, MCMC modeling of the HATSouth photometry, follow-up photometry, and the RV measurements. The methodology is described fully in Bakos et al. (2010). The resulting planetary parameters are set out in Table 7 . We list two sets of planet parameters: for the case of a circular orbit $(e=0)$ and for the case that eccentricity is allowed to vary, whereby we get a best-fit eccentricity of $e=0.25 \pm 0.10$.

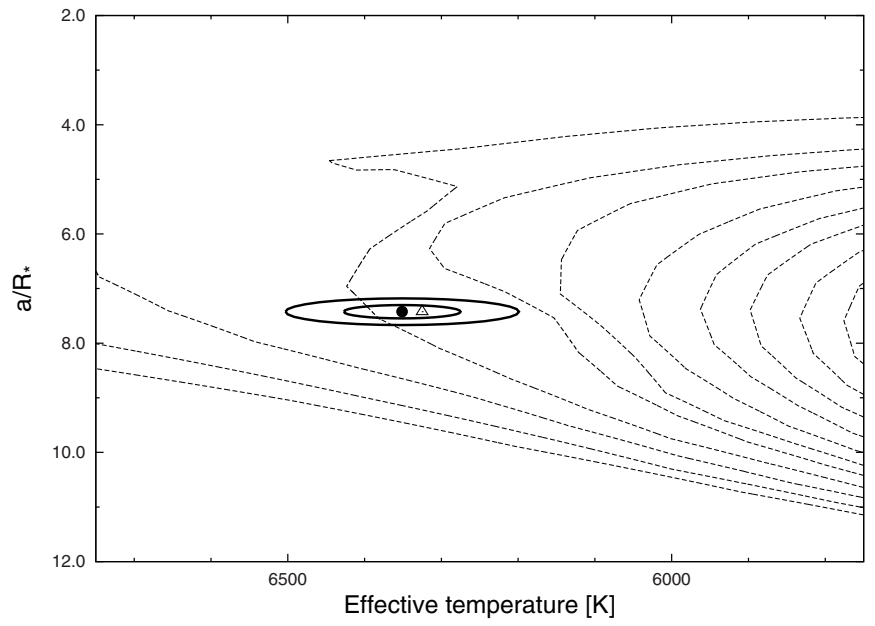

Figure 8. Model isochrones from Yi et al. (2001) for the measured metallicity of HATS $-3,[\mathrm{Fe} / \mathrm{H}]=-0.157$, and ages of $0.2 \mathrm{Gyr}$ (lowermost line) and $1-10 \mathrm{Gyr}$ in $1 \mathrm{Gyr}$ increments (left to right). The adopted values of $T_{\mathrm{eff} \star}$ and $a / R_{\star}$, as discussed in Section 3.1, are shown together with their $1 \sigma$ and $2 \sigma$ confidence ellipsoids. The open triangle shows the values from the initial SME iteration.

To estimate the significance of the non-zero eccentricity measurement, we use the Lucy \& Sweeney (1971) test, which determines whether the best-fit eccentric orbit is significant visá-vis the best-fit circular orbit at a $5 \%$ level. For HATS-3b we find a non-negligible (7\%) probability that the RV observations are consistent with a circular orbit, while the free-eccentricity and fixed-circular-orbit models yield nearly identical values for the Bayesian information criterion. We conclude that we cannot rule out a circular orbit based on the current RV observations.

\section{DISCUSSION}

HATS-3b is the third planet to be discovered as part of the ongoing HATSouth survey for transiting exoplanets. Although it is the least massive of the three exoplanets with $M_{p} \approx 1.07 M_{\mathrm{J}}$, the host star is the most massive of the three host stars $\left(M_{\star}=\right.$ $\left.1.21 M_{\odot}\right)$.

When we allow the eccentricity to depart from $e=0$ in the global fit, we find a high eccentricity $(e=0.253 \pm 0.110)$ for HATS-3b. However, like many planetary systems, the eccentricity is not well constrained by the RV measurements, and more data will be required before this high eccentricity can be confirmed.

The $v \sin i=9.12 \pm 1.31 \mathrm{~km} \mathrm{~s}^{-1}$, combined with the large radius of the planet $\left(R_{p}=1.381 \pm 0.035 R_{\mathrm{J}}\right)$, makes HATS-3b a prime target for follow-up Rossiter-McLaughlin monitoring to determine the spin-orbit alignment of the system. Interestingly, with $T_{\text {eff } \star}=6351 \pm 76 \mathrm{~K}$ this star lies just above the temperature of $6250 \mathrm{~K}$, which is noted as apparently marking a shift from aligned to misaligned hot Jupiters (Winn et al. 2010; Albrecht et al. 2012).

The power of the HATSouth network was illustrated in Penev et al. (2013), whereby three consecutive transits were detected at three different ground stations. For HATS-3b we show another aspect of the global network, that for much of the year observations from the sites give considerable overlap. Figure 9 shows that in pre-discovery a single transit of HATS$3 \mathrm{~b}$ was simultaneously monitored by two different telescopes located over $8000 \mathrm{~km}$ apart on the Earth. This is testament to an active, homogeneous, global network. 
Table 7

Orbital and Planetary Parameters

\begin{tabular}{|c|c|c|}
\hline Parameter & $\begin{array}{c}\text { Value } \\
\text { Circular }\end{array}$ & $\begin{array}{c}\text { Value } \\
\text { Eccentric }\end{array}$ \\
\hline \multicolumn{3}{|l|}{ Light curve parameters } \\
\hline$P$ (days) & $3.547851 \pm 0.000005$ & $3.547849 \pm 0.000005$ \\
\hline$T_{c}(\mathrm{BJD})^{\mathrm{a}}$ & $2456155.96734 \pm 0.00014$ & $2456092.10547 \pm 0.00039$ \\
\hline$T_{14}(\text { days })^{\mathrm{a}}$ & $0.1494 \pm 0.0007$ & $0.1486 \pm 0.0011$ \\
\hline$T_{12}=T_{34}(\text { days })^{\mathrm{a}}$ & $0.0177 \pm 0.0007$ & $0.0175 \pm 0.0007$ \\
\hline$a / R_{\star}$ & $7.42 \pm 0.12$ & $6.18_{-0.64}^{+0.88}$ \\
\hline$\zeta / R_{\star}^{\mathrm{b}}$ & $15.16 \pm 0.03$ & $15.22_{-0.06}^{+0.11}$ \\
\hline$R_{p} / R_{\star}$ & $0.1011 \pm 0.0006$ & $0.1010 \pm 0.0005$ \\
\hline$b \equiv a \cos i / R_{\star}$ & $0.497_{-0.027}^{+0.024}$ & $0.489_{-0.029}^{+0.024}$ \\
\hline$i\left(^{\circ}\right)$ & $86.2 \pm 0.3$ & $84.3_{-2.0}^{+1.3}$ \\
\hline \multicolumn{3}{|l|}{ Limb-darkening coefficients ${ }^{c}$} \\
\hline$a_{g}($ linear term $)$ & 0.4135 & 0.4160 \\
\hline$b_{g}$ (quadratic term) & 0.3301 & 0.3286 \\
\hline$a_{r}$ & 0.2592 & 0.2607 \\
\hline$b_{r}$ & 0.3725 & 0.3721 \\
\hline$a_{i}$ & 0.1903 & 0.1915 \\
\hline$b_{i}$ & 0.3599 & 0.3597 \\
\hline \multicolumn{3}{|l|}{ RV parameters } \\
\hline$K\left(\mathrm{~m} \mathrm{~s}^{-1}\right)$ & $125.7 \pm 15.7$ & $131.7 \pm 17.2$ \\
\hline$\sqrt{e} \cos \omega$ & $\ldots$ & $-0.355_{-0.089}^{+0.122}$ \\
\hline$\sqrt{e} \sin \omega$ & $\cdots$ & $0.356_{-0.252}^{+0.101}$ \\
\hline$e \cos \omega$ & $\cdots$ & $-0.176 \pm 0.080$ \\
\hline$e \sin \omega$ & $\cdots$ & $0.175 \pm 0.115$ \\
\hline$e$ & 0 & $0.253 \pm 0.110$ \\
\hline$\omega$ & $\ldots$ & $134 \pm 25$ \\
\hline CORALIE RV jitter $\left(\mathrm{m} \mathrm{s}^{-1}\right)^{\mathrm{d}}$ & 51.0 & 51.0 \\
\hline FEROS RV jitter $\left(\mathrm{m} \mathrm{s}^{-1}\right)$ & 9.5 & 9.5 \\
\hline CYCLOPS RV jitter $\left(\mathrm{m} \mathrm{s}^{-1}\right)$ & 65.0 & 65.0 \\
\hline \multicolumn{3}{|l|}{ Planetary parameters } \\
\hline$M_{p}\left(M_{\mathrm{J}}\right)$ & $1.071 \pm 0.136$ & $1.138 \pm 0.152$ \\
\hline$R_{p}\left(R_{\mathrm{J}}\right)$ & $1.381 \pm 0.035$ & $1.697 \pm 0.238$ \\
\hline$C\left(M_{p}, R_{p}\right)^{\mathrm{e}}$ & 0.15 & 0.24 \\
\hline$\rho_{p}\left(\mathrm{~g} \mathrm{~cm}^{-3}\right)$ & $0.51 \pm 0.07$ & $0.28_{-0.08}^{+0.18}$ \\
\hline $\log g_{p}(\mathrm{cgs})$ & $3.14 \pm 0.06$ & $2.99 \pm 0.12$ \\
\hline$a(\mathrm{AU})$ & $0.0485_{-0.0006}^{+0.0004}$ & $0.0497 \pm 0.0011$ \\
\hline$T_{\mathrm{eq}}(\mathrm{K})$ & $1648 \pm 24$ & $1816 \pm 127$ \\
\hline$\Theta^{f}$ & $0.062 \pm 0.008$ & $0.051_{-0.008}^{+0.012}$ \\
\hline$\langle F\rangle\left(10^{9} \mathrm{erg} \mathrm{s}^{-1} \mathrm{~cm}^{-2}\right)^{\mathrm{g}}$ & $1.66 \pm 0.10$ & $2.46_{-0.55}^{+0.90}$ \\
\hline
\end{tabular}

Notes.

${ }^{\text {a }} T_{c}$ : reference epoch of mid-transit that minimizes the correlation with the orbital period. BJD is calculated from UTC. $T_{14}$ : total transit duration, time between first to last contact; $T_{12}=T_{34}$ : ingress/egress time, time between first and second or third and fourth contact.

${ }^{\mathrm{b}}$ Reciprocal of the half-duration of the transit used as a jump parameter in our MCMC analysis in place of $a / R_{\star}$. It is related to $a / R_{\star}$ by the expression $\zeta / R_{\star}=a / R_{\star}(2 \pi(1+e \sin \omega)) /\left(P \sqrt{1-b^{2}} \sqrt{1-e^{2}}\right)$ (Bakos et al. 2010).

${ }^{c}$ Values for a quadratic law given separately for the Sloan $g, r$, and $i$ filters. These values were adopted from the tabulations by Claret (2004) according to the spectroscopic (SME) parameters listed in Table 6.

${ }^{d}$ This jitter was added in quadrature to the RV uncertainties for each instrument such that $\chi^{2} /$ dof $=1$ for the observations from that instrument for the model where eccentricity is allowed to vary from zero.

${ }^{\mathrm{e}}$ Correlation coefficient between the planetary mass $M_{p}$ and radius $R_{p}$ determined from the posterior distribution via $\left\langle\left(M_{p}-\left\langle M_{p}\right\rangle\right)\left(R_{p}-\left\langle R_{p}\right\rangle\right) /\left(\sigma_{M_{p}} \sigma_{R_{p}}\right)\right\rangle$, where $\langle\cdot\rangle$ is the expectation value operator and $\sigma_{x}$ is the standard deviation of parameter $x$.

${ }^{\mathrm{f}}$ The Safronov number is given by $\Theta=(1 / 2)\left(V_{\text {esc }} / V_{\text {orb }}\right)^{2}=\left(a / R_{p}\right)\left(M_{p} / M_{\star}\right)$ (see Hansen \& Barman 2007).

$\mathrm{g}$ Incoming flux per unit surface area, averaged over the orbit.

The large number of promising candidates emerging from the HATSouth network, typically at magnitudes of $10.5<r<$ 15.0 , necessitates a new approach to candidate follow-up, as it is not feasible to get high-resolution, multi-epoch data for all these candidates. Instead, we are finding that a single low- resolution ( $R$ 3000) spectrum is efficiently identifying giants, which contaminate our brightest candidates at the $35 \%$ level. Similarly, multi-epoch $R 7000$ spectra are proving an efficient means with which to rule out candidates with stellar mass companions, which contaminant the faint end of our candidates 


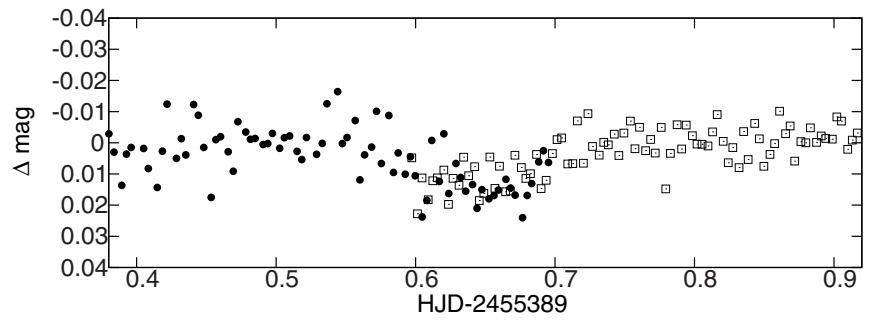

Figure 9. Single transit event from 2010 July 11 simultaneously observed from the HATSouth telescopes in Namibia (filled circles) and Chile (open squares). For 2 hr, both telescopes were monitoring HATS-3b in transit. Such simultaneous monitoring is possible for much of the year with the HATSouth network.

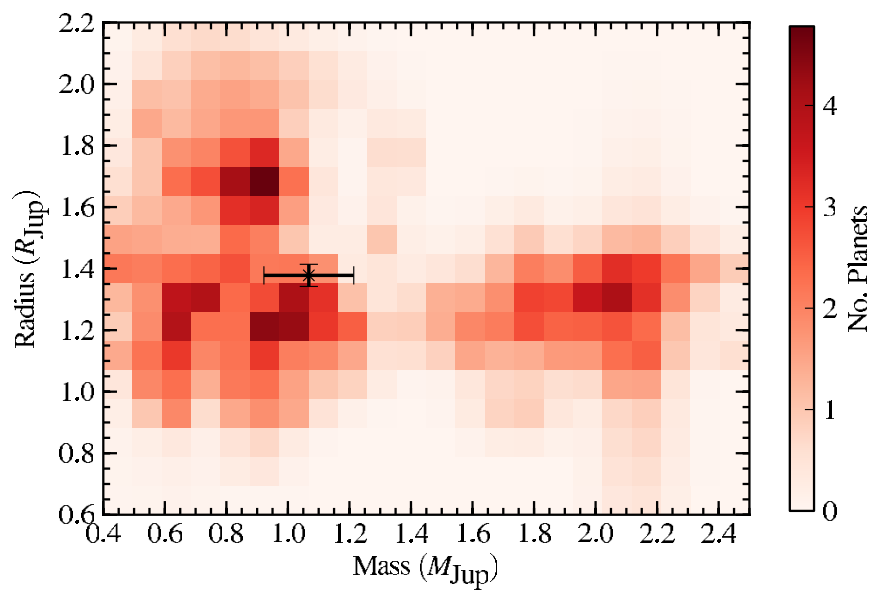

Figure 10. Density plot showing the mass and radius for published transiting hot Jupiters, defined as planets with $M_{p}>0.5 M_{\mathrm{J}}$ and $P<10$ days. HATS-3b is plotted in black with appropriate uncertainties.

(A color version of this figure is available in the online journal.)

at level of $27 \%$. With the number of transiting planet candidates from surveys only set to increase over the next five years, particularly with the planned TESS mission (Ricker et al. 2010), low-resolution reconnaissance as set out in this paper is a useful approach to take to candidate vetting.

There are 125 published transiting hot Jupiters (defined as $M_{p}>0.5 M_{\mathrm{J}}, P<10$ days) with both radius and mass precisely determined, ${ }^{13}$ so we can begin to plot the mass-radius diagram in terms of a density distribution of known exoplanets rather than just individual points. Such a plot is presented in Figure 10, with the position of HATS-3b marked in black with appropriate error bars. Interestingly, this diagram reveals an underdensity of planets at 1.2-1.6 $M_{\mathrm{J}}$, even though there is no selection bias against detecting planets in this mass range with transit surveys. While it is possible that the gap is simply a feature of small number statistics, it further supports the case for expanding the sample of hot Jupiters to probe global trends in the populations.

Development of the HATSouth project was funded by NSF MRI grant NSF/AST-0723074, operations are supported by NASA grant NNX09AB29G, and follow-up observations receive partial support from grant NSF/AST-1108686. Work at the Australian National University is supported by ARC Laureate Fellowship Grant FL0992131. Follow-up observations with the ESO $2.2 \mathrm{~m} /$ FEROS instrument were performed under MPI guaranteed time (P087.A-9014(A), P088.A-9008(A), P089.A-9008(A)) and Chilean time (P087.C-0508(A)). A.J.

13 http://exoplanets.org acknowledges support from Fondecyt project 1130857, Ministry of Economy ICM Nucleus P10-022-F, Anillo ACT-086, and BASAL CATA PGB-06. V.S. acknowledges support from BASAL CATA PFB-06. M.R. acknowledges support from FONDECYT postdoctoral fellowship No. 3120097. R.B. and N.E. acknowledge support from CONICYT-PCHA/Doctorado Nacional and Fondecyt project 1130857. This work is based on observations made with ESO Telescopes at the La Silla Observatory under program IDs P087.A-9014(A), P088.A-9008(A), P089.A-9008(A), P087.C-0508(A), and 089.A-9006(A). This paper also uses observations obtained with facilities of the Las Cumbres Observatory Global Telescope. Work at UNSW has been supported by ARC Australian Professorial Fellowship grant DP0774000, ARC LIEF grant LE0989347, and ARC Super Science Fellowships FS100100046. We acknowledge the use of the AAVSO Photometric All-Sky Survey (APASS), funded by the Robert Martin Ayers Sciences Fund, and the SIMBAD database, operated at CDS, Strasbourg, France. Operations at the MPG/ESO $2.2 \mathrm{~m}$ Telescope are jointly performed by the Max Planck Gesellschaft and the European Southern Observatory. The imaging system GROND has been built by the highenergy group of MPE in collaboration with the LSW Tautenburg and ESO. We thank Timo Anguita and Régis Lachaume for their technical assistance during the observations at the MPG/ESO $2.2 \mathrm{~m}$ Telescope.

\section{REFERENCES}

Albrecht, S., Winn, J. N., Johnson, J. A., et al. 2012, ApJ, 757, 18 Anderson, D. R., Collier Cameron, A., Gillon, M., et al. 2012, MNRAS, 422, 1988

Anderson, D. R., Gillon, M., Hellier, C., et al. 2008, MNRAS, 387, L4

Auvergne, M., Bodin, P., Boisnard, L., et al. 2009, A\&A, 506, 411

Bakos, G., Noyes, R. W., Kovács, G., et al. 2004, PASP, 116, 266

Bakos, G. Á., Csubry, Z., Penev, K., et al. 2013, PASP, 125, 154

Bakos, G. Á., Torres, G., Pál, A., et al. 2010, ApJ, 710, 1724

Bayliss, D. D. R., \& Sackett, P. D. 2011, ApJ, 743, 103

Bell, R. A., Edvardsson, B., \& Gustafsson, B. 1985, MNRAS, 212, 497

Berdyugina, S. V., \& Savanov, I. S. 1994, AstL, 20, 755

Bertin, E., \& Arnouts, S. 1996, A\&AS, 117, 393

Bessell, M. S. 1999, PASP, 111, 1426

Bessell, M. S. 2007, PASP, 119, 605

Borucki, W. J., Koch, D., Basri, G., et al. 2010, Sci, 327, 977

Cardelli, J. A., Clayton, G. C., \& Mathis, J. S. 1989, ApJ, 345, 245

Charbonneau, D., Brown, T. M., Noyes, R. W., \& Gilliland, R. L. 2002, ApJ, 568,377

Claret, A. 2004, A\&A, 428, 1001

de Medeiros, J. R., Silva, J. R. P., Do Nascimento, J. D., Jr., et al. 2006, A\&A, 458,895

Dopita, M., Hart, J., McGregor, P., et al. 2007, Ap\&SS, 310, 255

Fressin, F., Torres, G., Charbonneau, D., et al. 2013, ApJ, 766, 81

Greiner, J., Bornemann, W., Clemens, C., et al. 2008, PASP, 120, 405

Gustafsson, B., Edvardsson, B., Eriksson, K., et al. 2008, A\&A, 486, 951

Hamuy, M., Walker, A. R., Suntzeff, N. B., et al. 1992, PASP, 104, 533

Hansen, B. M. S., \& Barman, T. 2007, ApJ, 671, 861

Hartman, J. D., Bakos, G. Á., Torres, G., et al. 2011, ApJ, 742, 59

Hellier, C., Anderson, D. R., Collier Cameron, A., et al. 2010, ApJL, 723, L60

Hellier, C., Anderson, D. R., Gillon, M., et al. 2009, ApJL, 690, L89

Ibata, R. A., \& Irwin, M. J. 1997, AJ, 113, 1865

Kaufer, A., \& Pasquini, L. 1998, Proc. SPIE, 3355, 844

Knutson, H. A., Charbonneau, D., Allen, L. E., et al. 2007, Natur, 447, 183

Kovács, G., Bakos, G., \& Noyes, R. W. 2005, MNRAS, 356, 557

Kovács, G., Zucker, S., \& Mazeh, T. 2002, A\&A, 391, 369

Lucy, L. B., \& Sweeney, M. A. 1971, AJ, 76, 544

Mancini, L., Nikolov, N., Southworth, J., et al. 2013, MNRAS, 430, 2932

Mohler-Fischer, M., Mancini, L., Hartman, J. D., et al. 2013, A\&A, in press (arXiv:1304.2140)

Nidever, D. L., Marcy, G. W., Butler, R. P., Fischer, D. A., \& Vogt, S. S. 2002, ApJS, 141, 503

Penev, K., Bakos, G. Á., Bayliss, D., et al. 2013, AJ, 145, 5

Pollacco, D. L., Skillen, I., Collier Cameron, A., et al. 2006, PASP, 118, 1407 
Queloz, D., Anderson, D., Collier Cameron, A., et al. 2010, A\&A, 517, L1 Queloz, D., Eggenberger, A., Mayor, M., et al. 2000a, A\&A, 359, L13 Queloz, D., Mayor, M., Weber, L., et al. 2000b, A\&A, 354, 99

Ricker, G. R., Latham, D. W., Vanderspek, R. K., et al. 2010, BAAS, 42, 215

Robin, A. C., Reylé, C., Derrière, S., \& Picaud, S. 2003, A\&A, 409, 523

Robinson, S. E., Ammons, S. M., Kretke, K. A., et al. 2007, ApJS, 169, 430

Schlegel, D. J., Finkbeiner, D. P., \& Davis, M. 1998, ApJ, 500, 525
Southworth, J., Hinse, T. C., Jørgensen, U. G., et al. 2009, MNRAS, 396, 1023 Sozzetti, A., Torres, G., Charbonneau, D., et al. 2007, ApJ, 664, 1190 Stetson, P. B. 1987, PASP, 99, 191

Valenti, J. A., \& Piskunov, N. 1996, A\&AS, 118, 595

Wilson, D. M., Gillon, M., Hellier, C., et al. 2008, ApJL, 675, L113

Winn, J. N., Fabrycky, D., Albrecht, S., \& Johnson, J. A. 2010, ApJL, 718, L145 Yi, S., Demarque, P., Kim, Y.-C., et al. 2001, ApJS, 136, 417 